IJMMS 27:9 (2001) 541-546

PII. S0161171201006597

http://ijmms.hindawi.com

(c) Hindawi Publishing Corp.

\title{
ON SOLUTIONS OF THE GOŁABB-SCHINZEL EQUATION
}

\section{ANNA MUREŃKO}

(Received 29 January 2001)

\begin{abstract}
We determine the solutions $f:(0, \infty) \rightarrow[0, \infty)$ of the functional equation $f(x+$ $f(x) y)=f(x) f(y)$ that are continuous at a point $a>0$ such that $f(a)>0$. This is a partial solution of a problem raised by Brzdęk.
\end{abstract}

2000 Mathematics Subject Classification. 39B22.

The well-known Gołąb-Schinzel functional equation

$$
f(x+f(x) y)=f(x) f(y)
$$

has been studied by many authors (cf. $[1,3,5,7,10]$ ) in many classes of functions. Recently Aczél and Schwaiger [2], motivated by a problem of Kahlig, solved the following conditional version of (1)

$$
f(x+f(x) y)=f(x) f(y) \text { for } x \geq 0, y \geq 0,
$$

in the class of continuous functions $f: \mathbb{R} \rightarrow \mathbb{R}$, where $\mathbb{R}$ denotes the set of real numbers. Some further conditional generalizations of (1) have been considered by Reich [9] (see also [8] and Brzdęk [4]).

At the 38th International Symposium on Functional Equations (Noszvaj, Hungary, June 11-17, 2000) Brzdęk raised, among others, the problem (see [6]) of solving the equation

$$
f(x+f(x) y)=f(x) f(y), \quad \text { whenever } x, y, x+f(x) y \in \mathbb{R}_{+},
$$

in the class of functions $f: \mathbb{R}_{+} \rightarrow \mathbb{R}$ that are continuous at a point, where $\mathbb{R}_{+}=(0, \infty)$. We give a partial solution to the problem, namely we determine the solutions $f: \mathbb{R}_{+} \rightarrow$ $[0, \infty)$ of (3) that are continuous at a point $a \in \mathbb{R}_{+}$such that $f(a)>0$. Note that actually equations (1) and (3) have the same solutions in the class of functions $f: \mathbb{R}_{+} \rightarrow[0, \infty)$.

From now on we assume that $f: \mathbb{R}_{+} \rightarrow[0, \infty)$ is a solution of (3), continuous at a point $a \in \mathbb{R}_{+}$such that $f(a)>0$.

We start with some lemmas.

LEMmA 1. Suppose that $y_{2}>y_{1}>0$ and $f\left(y_{1}\right)=f\left(y_{2}\right)>0$. Then

(a) $f\left(t+\left(y_{2}-y_{1}\right)\right)=f(t)$ for $t \geq y_{1}$;

(b) for every $z>0$ such that $f(z)>0$,

$$
f\left(t+f(z)\left(y_{2}-y_{1}\right)\right)=f(t) \quad \text { for } t \geq z+y_{1} f(z) ;
$$


(c) if $z_{1}, z_{2}>0$ and $f\left(z_{2}\right)>f\left(z_{1}\right)>0$, then

$f\left(t+\left(f\left(z_{2}\right)-f\left(z_{1}\right)\right)\left(y_{2}-y_{1}\right)\right)=f(t) \quad$ for $t \geq \max \left\{z_{1}+y_{1} f\left(z_{1}\right), z_{2}+y_{1} f\left(z_{2}\right)\right\}$.

Proof. (a) We argue in the same way as in [2, 7]. Namely, for $t \geq y_{1}$, by (3) we have

$$
\begin{aligned}
f\left(t+\left(y_{2}-y_{1}\right)\right) & =f\left(y_{2}+\frac{t-y_{1}}{f\left(y_{1}\right)} f\left(y_{1}\right)\right)=f\left(y_{2}+\frac{t-y_{1}}{f\left(y_{1}\right)} f\left(y_{2}\right)\right) \\
& =f\left(y_{2}\right) f\left(\frac{t-y_{1}}{f\left(y_{1}\right)}\right)=f\left(y_{1}\right) f\left(\frac{t-y_{1}}{f\left(y_{1}\right)}\right) \\
& =f\left(y_{1}+\frac{t-y_{1}}{f\left(y_{1}\right)} f\left(y_{1}\right)\right)=f(t) .
\end{aligned}
$$

(b) For every $z>0$ such that $f(z)>0$ we have

$$
f\left(z+y_{1} f(z)\right)=f(z) f\left(y_{1}\right)=f(z) f\left(y_{2}\right)=f\left(z+y_{2} f(z)\right)
$$

and consequently by (a) (with $y_{1}$ and $y_{2}$ replaced by $z+y_{1} f(z)$ and $z+y_{2} f(z)$ )

$$
f(t)=f\left[t+\left(z+y_{2} f(z)-z-y_{1} f(z)\right)\right]=f\left(t+f(z)\left(y_{2}-y_{1}\right)\right)
$$

for $t \geq z+y_{1} f(z)$.

(c) Since $\left(f\left(z_{2}\right)-f\left(z_{1}\right)\right)\left(y_{2}-y_{1}\right)>0, t+\left(f\left(z_{2}\right)-f\left(z_{1}\right)\right)\left(y_{2}-y_{1}\right) \geq \max \left\{z_{1}+\right.$ $\left.y_{1} f\left(z_{1}\right), z_{2}+y_{1} f\left(z_{2}\right)\right\}$ for $t \geq \max \left\{z_{1}+y_{1} f\left(z_{1}\right), z_{2}+y_{1} f\left(z_{2}\right)\right\}$. Thus using (b) twice, for $z=z_{1}$ and $z=z_{2}$ (the first time with $t$ replaced by $t+\left(f\left(z_{2}\right)-f\left(z_{1}\right)\right)\left(y_{2}-y_{1}\right)$ ), we have

$$
\begin{aligned}
f(t+ & \left.\left(f\left(z_{2}\right)-f\left(z_{1}\right)\right)\left(y_{2}-y_{1}\right)\right) \\
& =f\left[t+\left(f\left(z_{2}\right)-f\left(z_{1}\right)\right)\left(y_{2}-y_{1}\right)+f\left(z_{1}\right)\left(y_{2}-y_{1}\right)\right] \\
& =f\left(t+f\left(z_{2}\right)\left(y_{2}-y_{1}\right)\right)=f(t)
\end{aligned}
$$

for $t \geq \max \left\{z_{1}+y_{1} f\left(z_{1}\right), z_{2}+y_{1} f\left(z_{2}\right)\right\}$.

LEMMA 2. Let $y_{2}>y_{1}>0$ and $f\left(y_{1}\right)=f\left(y_{2}\right)>0$. Then there exists $x_{0}>0$ such that for every $d>0$ there is $c \in(0, d)$ with $f(t+c)=f(t)$ for $t \geq x_{0}$.

Proof. First suppose that there is a neighbourhood $U=(a-\delta, a+\delta)$ of $a$ on which $f$ is constant. Then for every $x \in U$ such that $a<x$, from Lemma 1 (a), we get

$$
f(t+(x-a))=f(t) \text { for } t \geq a .
$$

Thus it is enough to take $x_{0}=a$.

Now assume that there does not exist any neighbourhood of $a$ on which $f$ is constant. Take $\varepsilon \in(0, f(a))$. The continuity of $f$ at $a$ implies that there exists $\delta \in(0,1)$ such that for every $x \in U_{1}=(a-\delta, a+\delta)$ we have $f(x) \in(f(a)-\varepsilon, f(a)+\varepsilon)$. Take $x_{1}, x_{2} \in U_{1}$ such that $f\left(x_{1}\right)<f\left(x_{2}\right)$. Then $f\left(x_{2}\right)-f\left(x_{1}\right)<2 \varepsilon$. From $\varepsilon<f(a)$ we infer $f\left(x_{1}\right)>0$ and by Lemma 1 (c) we get

$$
f\left(t+\left(f\left(x_{2}\right)-f\left(x_{1}\right)\right)\left(y_{2}-y_{1}\right)\right)=f(t) \text { for } t \geq \max \left\{x_{1}+y_{1} f\left(x_{1}\right), x_{2}+y_{1} f\left(x_{2}\right)\right\} \text {. }
$$

Next by a suitable choice of $\varepsilon$ the value $c:=\left(f\left(x_{2}\right)-f\left(x_{1}\right)\right)\left(y_{2}-y_{1}\right)$ can be made arbitrarily small. Moreover, $x_{1}, x_{2}<a+1$ and $f\left(x_{1}\right), f\left(x_{2}\right)<f(a)+\varepsilon<2 f(a)$, which means that $f(t+c)=f(t)$ for $t \geq x_{0}:=a+1+y_{1} 2 f(a)$. This completes the proof. 
LEMMA 3. If for some $y_{2}>y_{1}>0, f\left(y_{1}\right)=f\left(y_{2}\right)>0$, then for every $\varepsilon>0$ and $e>0$ there is $c \in(0, e)$ with $f(t+c)=f(t)$ for $t \geq \varepsilon$.

Proof. By Lemma 2 there exists $x_{0}>0$ such that for arbitrarily small $c>0$

$$
f(t+c)=f(t) \text { for } t \geq x_{0} .
$$

By induction, from Lemma 1 (a), we get $f\left(y_{1}\right)=f\left(y_{1}+n\left(y_{2}-y_{1}\right)\right)$ for any positive integer $n$. Consequently there exists $x_{1} \in\left[x_{0}, \infty\right)$ with $f\left(x_{1}\right)=f\left(y_{1}\right)$.

Put $B=\left\{x>x_{0}: f(x)>0\right\}$. Clearly $x_{1} \in B$. Thus (12) implies that $B \cap A \neq \varnothing$ for every nontrivial interval $A \subset\left[x_{0}, \infty\right)$. Define a function $f_{1}:[0, \infty) \rightarrow\left[x_{0}, \infty\right)$ by

$$
f_{1}(x)=x_{1}+x f\left(x_{1}\right) .
$$

Note that $f_{1}$ is increasing. Let $\varepsilon>0$ and $y_{0} \in B \cap\left(f_{1}(0), f_{1}(\varepsilon)\right) \neq \varnothing$. By the continuity of $f_{1}$ there exists $z_{0} \in(0, \varepsilon)$ such that $f_{1}\left(z_{0}\right)=y_{0}$. Take $d>0$ with $f(t+d)=f(t)$ for $t \geq x_{0}$. Then

$$
f\left(y_{0}\right)=f\left(y_{0}+d\right) \neq 0 .
$$

The form of the function $f_{1}$ implies that there exists $z_{1}>z_{0}$ such that $f_{1}\left(z_{1}\right)=y_{0}+d$. Note that (14) yields

$$
\begin{aligned}
f\left(x_{1}\right. & \left.+z_{0} f\left(x_{1}\right)\right)=f\left(f_{1}\left(z_{0}\right)\right) \\
& =f\left(y_{0}\right)=f\left(y_{0}+d\right)=f\left(f_{1}\left(z_{1}\right)\right) \\
& =f\left(x_{1}+z_{1} f\left(x_{1}\right)\right) \neq 0 .
\end{aligned}
$$

Further by (3)

$$
f\left(x_{1}\right) f\left(z_{0}\right)=f\left(x_{1}\right) f\left(z_{1}\right) \neq 0,
$$

and consequently $f\left(z_{0}\right)=f\left(z_{1}\right)>0$. Hence, in view of Lemma $1(\mathrm{a})$, we infer that

$$
f\left(t+\left(z_{1}-z_{0}\right)\right)=f(t) \text { for } t \geq z_{0} .
$$

This completes the proof, because $\varepsilon>z_{0}$ and, choosing sufficiently small $d$, we can make $c:=\left(z_{1}-z_{0}\right)$ arbitrarily small.

LEMMA 4. If there exist $y_{2}>y_{1}>0$ such that $f\left(y_{1}\right)=f\left(y_{2}\right)>0$, then $f \equiv 1$.

Proof. First we show that $f(x)=f(a)=: b$ for $x \in \mathbb{R}_{+}$. For the proof by contradiction suppose that there exists $t_{0}>0$ with $f\left(t_{0}\right) \neq f(a)$. Put

$$
\varepsilon_{0}=\left|f\left(t_{0}\right)-f(a)\right|
$$

The continuity of $f$ at $a$ implies that there exists $\delta>0$ such that if $|x-a|<\delta$ then $|f(x)-f(a)|<\varepsilon_{0}$. By Lemma 3 there exists $y_{0}>0$ such that $\left|y_{0}-a\right|<\delta$ and $f\left(y_{0}\right)=$ $f\left(t_{0}\right)$, which means that $\left|f\left(t_{0}\right)-f(a)\right|<\varepsilon_{0}$, contrary to (18). Thus we have proved that $f \equiv b$. Clearly from (3) we get $b=f(a)=f(a+a f(a))=f(a)^{2}=b^{2}$ and consequently $b=1$. This completes the proof.

LEMMA 5. If $f$ is nonconstant then $(f(x)-1) / x$ is constant for all $x>0$ with $f(x)>0$. 
Proof. Suppose that $x>0, y>0, x \neq y, f(x) f(y)>0$, and

$$
\frac{f(x)-1}{x} \neq \frac{f(y)-1}{y} \text {. }
$$

Then $x+y f(x) \neq y+x f(y)$ and

$$
f(x+y f(x))=f(x) f(y)=f(y+x f(y))>0 .
$$

Thus, by Lemma $4, f \equiv 1$, a contradiction.

REMARK 6. If we denote the constant in Lemma 5 by $c$, then from Lemma 5 we get $f(x) \in\{c x+1,0\}$ for every $x>0$. In the case $c<0$ we have $f(x)=0$ for every $x \geq-1 / c$ (because $f \geq 0$ ).

LEMMA 7. Suppose that $f$ is nonconstant. Then,

(a) in the case $c:=(f(a)-1) / a<0, f(x)=c x+1$ for $x \in(0,-1 / c)$;

(b) in the case $c:=(f(a)-1) / a>0, f(x)=c x+1$ for $x>0$.

Proof. The continuity of $f$ at $a$ implies that there exists $\delta \in(0, a)$ such that $f(x)>0$ for every $x \in U=[a-\delta, a+\delta]$. Thus, by Remark 6, $f(x)=c x+1$ for $x \in U$.

Let $I=(a,-1 / c)$ if $c<0$ and $I=(a, \infty)$ if $c>0$. Put $B_{1}:=\{x \in(0, a): f(x)=0\}$, $B_{2}:=\{x \in I: f(x)=0\}, B=B_{1} \cup B_{2}$,

$$
d_{1}:=\left\{\begin{array}{ll}
\sup B_{1} & \text { if } B_{1} \neq \varnothing, \\
a-\delta & \text { if } B_{1}=\varnothing,
\end{array} \quad d_{2}:= \begin{cases}\inf B_{2} & \text { if } B_{2} \neq \varnothing, \\
a+\delta & \text { if } B_{2}=\varnothing .\end{cases}\right.
$$

Clearly $f(x)>0$ on the interval $A=\left(d_{1}, d_{2}\right) \supset(a-\delta, a+\delta)$.

(a) For the proof by contradiction suppose that there exists $b_{1} \in(0,-1 / c)$ with $f\left(b_{1}\right)=0$. Notice that $d_{2}<-1 / c$. Indeed, if $B_{2} \neq \varnothing$ then, since $B_{2} \subset(a,-1 / c)$, so $\inf B_{2}<-1 / c$. If not, then from Remark 6 we have that $a+\delta<-1 / c$. Consequently $d_{2}<-1 / c$. Thus $c d_{2}>-1$ and consequently $\delta+\delta c d_{2}>0$. Take $b \in B$ and $z \in A$ such that $|z-b|<\delta+\delta c d_{2}$. Define functions $h, g: U \rightarrow \mathbb{R}$ by

$$
\begin{array}{ll}
h(x)=x+z f(x) & \text { for } x \in U, \\
g(x)=x+b f(x) & \text { for } x \in U .
\end{array}
$$

By the continuity of $f$ on $U, h$ is continuous. Next, since $z<d_{2}$, so $c z>c d_{2}$ and $\delta+\delta c z>\delta+\delta c d_{2}>0$. Hence

$$
\begin{aligned}
& h(a)-h(a-\delta)=a+z(c a+1)-a+\delta-z[c(a-\delta)+1]=\delta+\delta c z>0, \\
& h(a+\delta)-h(a)=a+\delta+z[c(a+\delta)+1]-a-z(c a+1)=\delta+\delta c z>0 .
\end{aligned}
$$

Moreover $1>c a+1=f(a)>0$, whence

$$
\begin{aligned}
& |h(a)-g(a)|=|a+z(c a+1)-a-b(c a+1)| \\
& \quad=|z-b||c a+1|<|z-b|<\delta+\delta c d_{2}<\delta+\delta c z .
\end{aligned}
$$

From (23) and (24) we obtain

$$
h(a-\delta)<g(a)<h(a+\delta) .
$$


The continuity of $h$ implies that there exists $x_{0} \in(a-\delta, a+\delta)$ such that $h\left(x_{0}\right)=g(a)$. Since $a, x_{0}, z \in A$ and $b \in B$, so we have

$$
\begin{aligned}
0 & \neq f\left(x_{0}\right) f(z)=f\left(x_{0}+z f\left(x_{0}\right)\right)=f\left(h\left(x_{0}\right)\right) \\
& =f(g(a))=f(a+b f(a))=f(a) f(b)=0 .
\end{aligned}
$$

This contradiction ends the proof of (a).

(b) For the proof by contradiction suppose that $f\left(b_{1}\right)=0$ for some $b_{1}>0$. Since $c a+1=f(a)>0$, there are $b \in B$ and $z \in A$ such that $|z-b|<\delta /(c a+1)$. Define functions $h, g: U \rightarrow \mathbb{R}$ in the same way as in the proof of (a). Then (23) holds and

$$
|h(a)-g(a)|=|z-b||c a+1|<\frac{\delta}{c a+1}(c a+1)=\delta<\delta+\delta c z .
$$

Hence

$$
h(a-\delta)<g(a)<h(a+\delta) .
$$

We obtain a contradiction in a similar way as in the proof of (a).

LEMMA 8. If $c:=(f(a)-1) / a=0$, then $f(x)=1$ for $x>0$.

Proof. The continuity of $f$ at $a$ implies that there exists $\delta>0$ such that $f(x)>0$ for every $x \in[a-\delta, a+\delta]$. Thus, by Lemma 5 and Remark $6, f(x)=c x+1=1$ for every $x \in[a-\delta, a+\delta]$. Hence Lemma 4 implies that $f(x)=1$ for every $x>0$.

Finally from Lemmas 7 and 8 and Remark 6 we get the following theorem.

THEOREM 9. If a function $f: \mathbb{R}_{+} \rightarrow[0, \infty)$ is continuous at a point a such that $f(a) \neq 0$ and satisfies (3), then

$$
f(x)=\max \{c x+1,0\} \quad \forall x \in \mathbb{R}_{+} .
$$

ACKNOWLEDGement. I wish to thank Professor Janusz Brzdẹk for paying my attention to the problem and for his most valuable suggestions during the preparation of this paper.

\section{REFERENCES}

[1] J. Aczél and S. Gołạb, Remarks on one-parameter subsemigroups of the affine group and their homo- and isomorphisms, Aequationes Math. 4 (1970), 1-10. MR 41\#8561. Zbl 205.14802.

[2] J. Aczél and J. Schwaiger, Continuous solutions of the Gotab-Schinzel equation on the nonnegative reals and on related domains, Sb. Öster. Akad. Wiss. 208 (1999), 171177.

[3] K. Baron, On the continuous solutions of the Gotab-Schinzel equation, Aequationes Math. 38 (1989), no. 2-3, 155-162. MR 90i:39010. Zbl 702.39005.

[4] J. Brzdęk, On continuous solutions of a conditional Gołąb-Schinzel equation, to appear in Sb. Öster. Akad. Wiss.

[5] _ Subgroups of the group $Z_{n}$ and a generalization of the Gotab-Schinzel functional equation, Aequationes Math. 43 (1992), no. 1, 59-71. MR 93b:39006.

[6] _ 28. Problem, Report of Meeting: The 38th International Symposium on Functional Equations (Noszvaj, Hungary), June 11-18, 2000, Aequationes Math., in print.

[7] S. Gołąb and A. Schinzel, Sur l'équation fonctionnelle $f[x+y \cdot f(x)]=f(x) \cdot f(y)$, Publ. Math. Debrecen 6 (1959), 113-125 (French). MR 21\#5828. Zbl 083.35004. 
[8] L. Reich, Über die stetigen Lösungen der Gołab-Schinzel-Gleichung auf $\mathbb{R}$ und auf $\mathbb{R}_{\geq 0}$, to appear in Sb. Öster. Akad. Wiss.

[9] _ Über die stetigen Lösungen der Gołąb-Schinzel-Gleichung auf $\mathbb{R}_{\geq 0}$, Sb. Öster. Akad. Wiss. 208 (1999), 165-170 (German).

[10] S. Wołodźko, Solution générale de l'équation fonctionnelle $f[x+y f(x)]=f(x) f(y)$, Aequationes Math. 2 (1968), 12-29 (French). MR 38\#1422. Zbl 162.20402.

Anna Mureńko: Department of Mathematics, Pedagogical University, Rejtana 16 A, 35-310 RzESzów, POLAND

E-mail address: amurenko@poczta.onet.p1 


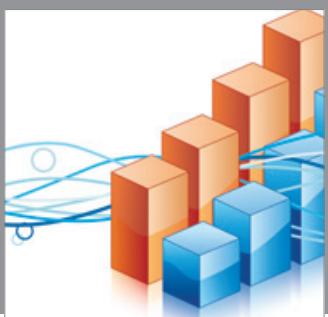

Advances in

Operations Research

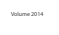

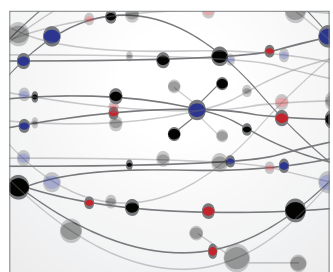

\section{The Scientific} World Journal
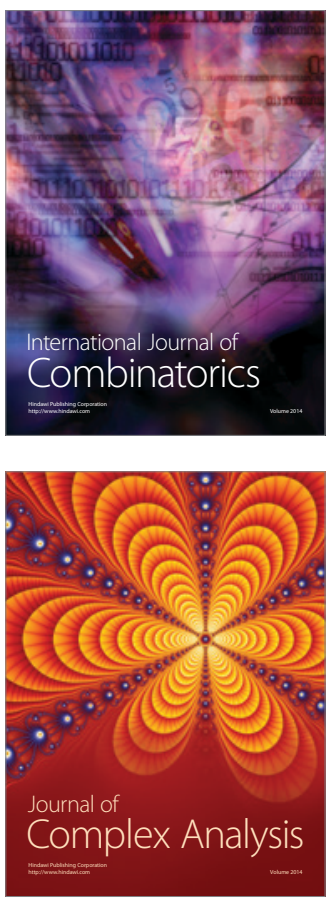

International Journal of

Mathematics and

Mathematical

Sciences
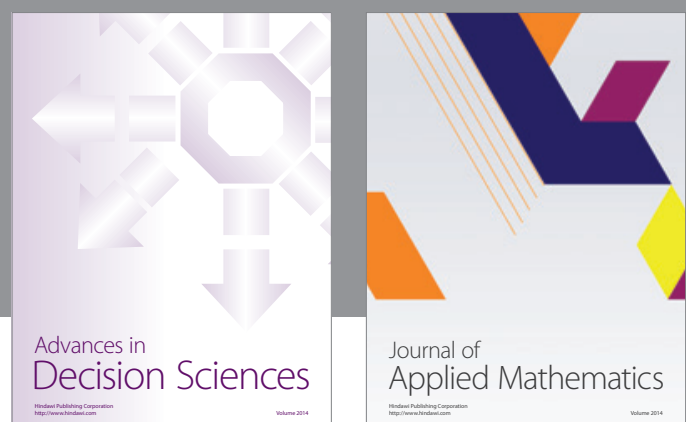

Journal of

Applied Mathematics
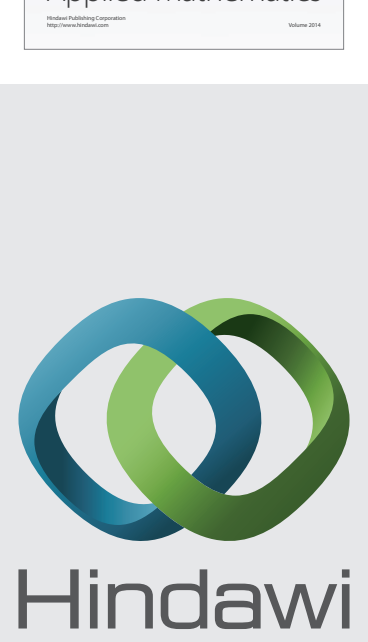

Submit your manuscripts at http://www.hindawi.com
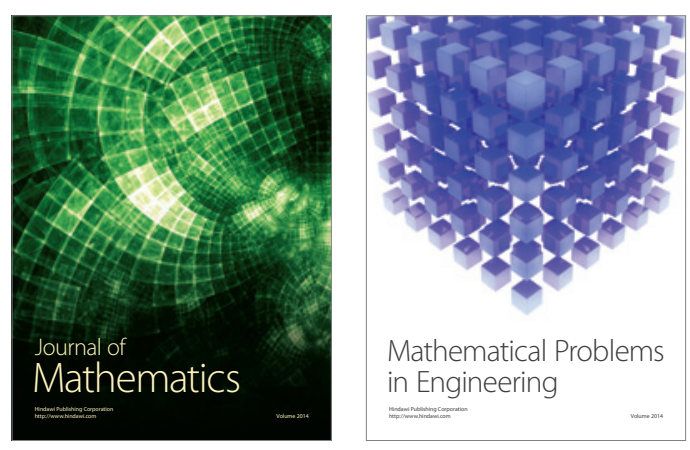

Mathematical Problems in Engineering
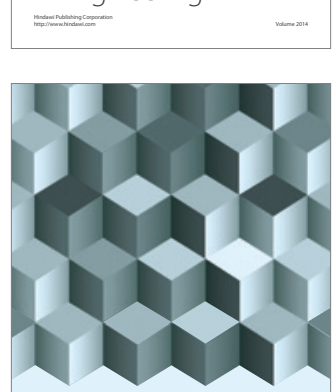

Journal of

Function Spaces
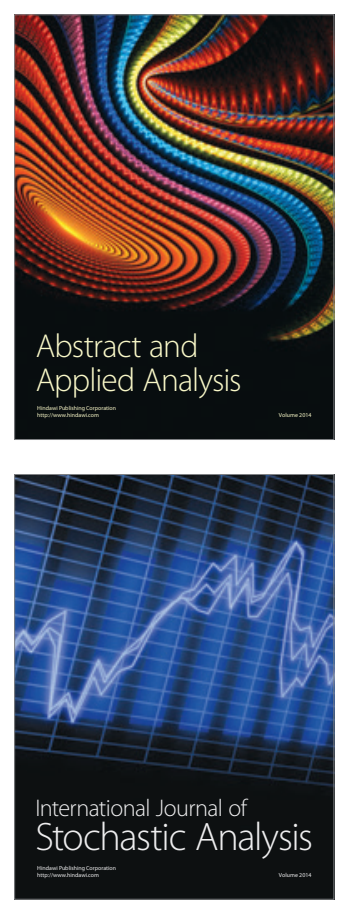

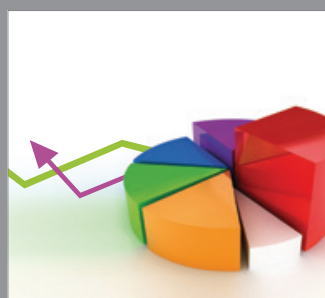

ournal of

Probability and Statistics

Promensencen
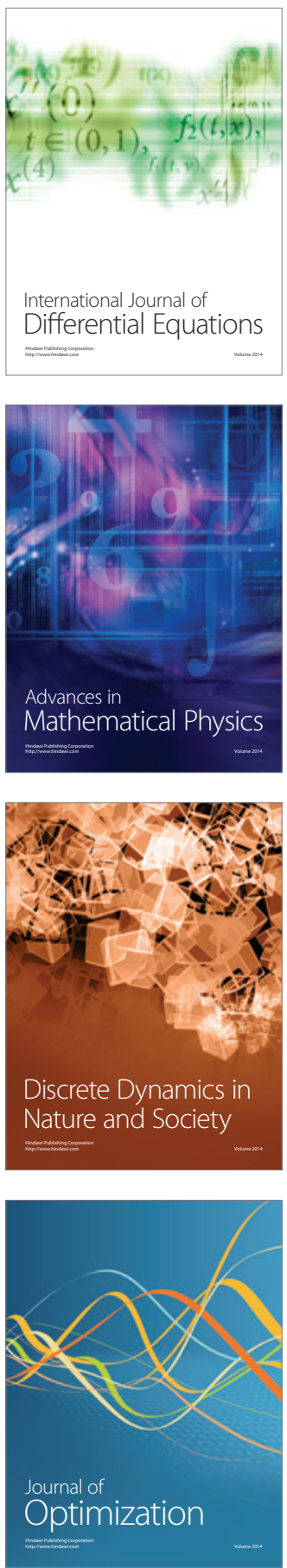